\title{
Identification of transcription factors MYC and C/EBP $\beta$ mediated regulatory networks in heart failure based on gene expression omnibus datasets
}

\author{
Haiwei Wang ${ }^{\dagger}$, Xinrui Wang ${ }^{\dagger}$, Liangpu Xu and Hua Cao
}

\begin{abstract}
Background: Heart failure is one of leading cause of death worldwide. However, the transcriptional profiling of heart failure is unclear. Moreover, the signaling pathways and transcription factors involving the heart failure development also are largely unknown. Using published Gene Expression Omnibus (GEO) datasets, in the present study, we aim to comprehensively analyze the differentially expressed genes in failing heart tissues, and identified the critical signaling pathways and transcription factors involving heart failure development.

Methods: The transcriptional profiling of heart failure was identified from previously published gene expression datasets deposited in GSE5406, GSE16499 and GSE68316. The enriched signaling pathways and transcription factors were analyzed using Database for Annotation, Visualization and Integrated Discovery (DAVID) website and gene set enrichment analysis (GSEA) assay. The transcriptional networks were created by Cytoscape.

Results: Compared with the normal heart tissues, 90 genes were particularly differentially expressed in failing heart tissues, and those genes were associated with multiple metabolism signaling pathways and insulin signaling pathway. Metabolism and insulin signaling pathway were both inactivated in failing heart tissues. Transcription factors MYC and C/EBP $\beta$ were both negatively associated with the expression profiling of failing heart tissues in GSEA assay. Moreover, compared with normal heart tissues, MYC and C/EBP $\beta$ were down regulated in failing heart tissues. Furthermore, MYC and C/EBP $\beta$ mediated downstream target genes were also decreased in failing heart tissues. MYC and C/EBP $\beta$ were positively correlated with each other. At last, we constructed MYC and C/EBP $\beta$ mediated regulatory networks in failing heart tissues, and identified the MYC and C/EBP $\beta$ target genes which had been reported involving the heart failure developmental progress.
\end{abstract}

Conclusions: Our results suggested that metabolism pathways and insulin signaling pathway, transcription factors MYC and C/EBP $\beta$ played critical roles in heart failure developmental progress.

Keywords: Heart failure, C/EBP $\beta$, MYC, Networks, Metabolism signaling pathway, Insulin signaling pathway

\footnotetext{
* Correspondence: caohua69@fjmu.edu.cn

${ }^{\dagger}$ Haiwei Wang and Xinrui Wang contributed equally to this work. Fujian Maternity and Child Health Hospital, Affiliated Hospital of Fujian Medical University, FuZhou 350001, FuJian, China
}

C The Author(s). 2020 Open Access This article is licensed under a Creative Commons Attribution 4.0 International License, which permits use, sharing, adaptation, distribution and reproduction in any medium or format, as long as you give appropriate credit to the original author(s) and the source, provide a link to the Creative Commons licence, and indicate if changes were made. The images or other third party material in this article are included in the article's Creative Commons licence, unless indicated otherwise in a credit line to the material. If material is not included in the article's Creative Commons licence and your intended use is not permitted by statutory regulation or exceeds the permitted use, you will need to obtain permission directly from the copyright holder. To view a copy of this licence, visit http://creativecommons.org/licenses/by/4.0/. The Creative Commons Public Domain Dedication waiver (http://creativecommons.org/publicdomain/zero/1.0/) applies to the data made available in this article, unless otherwise stated in a credit line to the data. 


\section{Background}

Heart failure is a rapidly growing public health issue and one of leading cause of death [1]. Serial vicious cycles of cardiomyocyte depletion, cardiac dilatation and mechanical dysfunction are culminating in heart failure [2]. Once patients have developed to the end stage of heart failure, intervention is limited to heart transplantation [3]. In order to understand the molecular mechanisms regulating heart failure, several studies have used microarrays for genome wide analysis of heart failure [4-6]. Transcriptional genomics results revealed that FOX families of transcription factors were associated with human heart failure [4]. The different mRNA splicing [5] and long non-coding RNA (lncRNA) [6] in diseased hearts was also comprehensively studied using gene microarrays. However, due to the complexity of genetic and epigenetic abnormality of heart failure, the previously reported gene expression signature in failing heart tissues is varied considerably from study to study, making it difficult to reconcile their findings or reach any definite conclusions [7]. Moreover, the mis-regulated molecular signaling pathways and key transcription factors in heart failure are largely unknown.

Transcription factors control the transcriptional activity of multiple target genes by binding to a specific region of the DNA sequence [8]. It has been reported that transcription factor C/EBP $\beta$ plays central roles in physiologic hypertrophy and heart failure [9]. C/EBP $\beta$ could repress cardiomyocyte growth and proliferation. Reducing C/EBP $\beta$ expression exaggerates the cardiac failure upon pressure overload [9]. TP53 is another major transcription factor in cardiac transcriptional network [10]. TP53 deficient hearts are resistant to the failure development upon acute pressure overload [11]. Interestingly, both $\mathrm{C} / \mathrm{EBP} \beta$ and TP53 are involving tumor developmental progress by regulating metabolism $[12,13]$ and TGF $\beta$ signaling pathway $[14,15]$.

MYC is an oncogene. High level of MYC expression is required for tumor initiation, progression and maintenance [16]. MYC regulates multiple critical cellular functions, for example, metabolism [17] and RNA splicing [18]. Inhibition of MYC by BET bromodomain inhibitor is a promising anti-cancer strategy [19]. Interestingly, transcriptional pause release in heart failure was mediated by BET bromodomain [20], and BET bromodomain inhibitors could suppresses the development of heart failure by the regulation of the innate inflammatory network [21]. All those results suggest the potentially significant roles of MYC in heart failure. However, the expression of MYC and MYC mediated downstream target genes are not studied in failing heart patients.

In the present study, using published GEO datasets, we tried to identify the signaling pathways and transcription factors associated with heart failure. We also tried to determine the MYC and C/EBP $\beta$ mediated downstream target genes and construct the complex transcriptional networks regulated by MYC and C/EBP $\beta$ in heart failure developmental progress.

\section{Methods \\ Data collection}

Gene expression series matrix of failing heart tissues and normal heart tissues was downloaded from GEO website (https://www.ncbi.nlm.nih.gov/geo/) with GEO number GSE5406, GSE16499 and GSE68316.

\section{GEO data processing}

All the expression datasets were processed separately using R software (version 3.5.0; https://www.r-project. $\operatorname{org} /)$. The matrix file of each dataset was annotated with corresponding platform. A probe was removed if it was not corresponded gene symbol, and the expression values were averaged if multiple probes corresponded to the same gene symbol using $\mathrm{R}$ software "plyr" package (version 1.8.5; https://cran.r-project.org/web/packages/ plyr/index.html). Plyr package includes multiple tools for splitting, applying and combining data. The different gene expression between failing heart tissues and normal heart tissues was determined using Student's t test.

\section{Venn diagrams}

Venn diagrams were generated using VENNY 2.1 software (http://bioinfogp.cnb.csic.es/tools/venny/index.html). VENNY 2.1 is an interactive tool for comparing lists.

\section{Kyoto encyclopedia of gens and genomes (KEGG) signaling pathway enrichment analysis}

KEGG signaling pathways and transcription factors analysis was performed using The Database for Annotation, Visualization and Integrated Discovery (DAVID) website (version 6.8; https://david.ncifcrf.gov) [22]. DAVID is a functional annotation tool for list of genes. Enrichment $P$-value and Benjamini false discovery rate (FDR) were generated. Enriched signaling pathways and transcription factors with $P$-value $<0.05$ was considered to be statistical significant.

\section{Gene set enrichment analysis (GSEA)}

GSEA was performed using GSEA 2.0 software [23]. Signaling pathways gene sets and transcription factor targets gene sets were downloaded from the GSEA Web site (http://www.broad.mit.edu/gsea/index.html). Genes ranked by signal-to-noise ratio, and statistical significance was determined by 1000 gene set permutations. The results of significance should meet the criteria of $\mathrm{P}$-value $<0.05$. 


\section{Heatmap presentation}

Heatmaps were created by R software "pheatmap" package (version 1.0.12; https://cran.r-project.org/web/packages/pheatmap/). "pheatmap" is a $\mathrm{R}$ package offering more dimensions and appearance of heatmaps. The clustering scale was determined by "average" method. The clustering distance was determined by the 'correlation' method. Other parameters were provided in the usage of the "pheatmap".

\section{Spearman correlation}

Spearman correlation was used to study the correlation between C/EBP $\beta$ and MYC expression by the " $\mathrm{lm}$ " method of R software. " $\mathrm{lm}$ " method was used for linear regression analysis in R. $P$-value $<0.05$ suggested the significant correlation between C/EBP $\beta$ and MYC expression.

\section{C/EBP $\beta$ and MYC associated transcriptional network}

The networks of C/EBP $\beta$ and MYC downstream target genes were created by Cytoscape GeneMANIA App. Cytoscape is an open source software platform for constructing complex networks and could be download from Cytoscape website (https://cytoscape.org/). Node degrees represent the power of the connection between the selected genes.

\section{Statistical analysis}

The box plots were generated from GraphPad software Prism8. GraphPad Prism8 was provided by GraphPad
Company (https://www.graphpad.com/). Statistical analysis was performed using the two-tailed paired Student's $\mathrm{t}$ test using $\mathrm{R}$ software. $\mathrm{R}$ software (version 3.5.0) was provided by The R Project (https://www.r-project.org/). $P$ value less than 0.05 was chosen to be statistically significant difference.

\section{Results}

\section{The transcriptomic features of heart failure}

To identify the differentially expressed genes and the critical signaling pathways and transcription factors during the development of heart failure, we analyzed the expression data of failing heart and normal heart tissues from previously published GEO datasets GSE5460 [4], GSE16499 [5] and GSE68316 [6]. Totally, 252 samples were collected, including 36 normal heart tissues and 216 failing heart tissues. The search strategies used for accessing the gene datasets were described in the flowchart (Fig. 1).

First, we analyzed the globe expression profiling of failing heart tissues in each dataset. Compared with the normal heart tissues, the differentially expressed genes in failing heart tissues $(P<0.01)$ were selected for further studies. This resulted in the identification of 2184 differentially expressed genes in GSE5406, 1644 differentially expressed genes in GSE16499 and 3477 differentially expressed genes in GSE68316 dataset (Fig. 2a). Among all the differentially expressed genes, only 4 genes were commonly up regulated and 86 genes were commonly

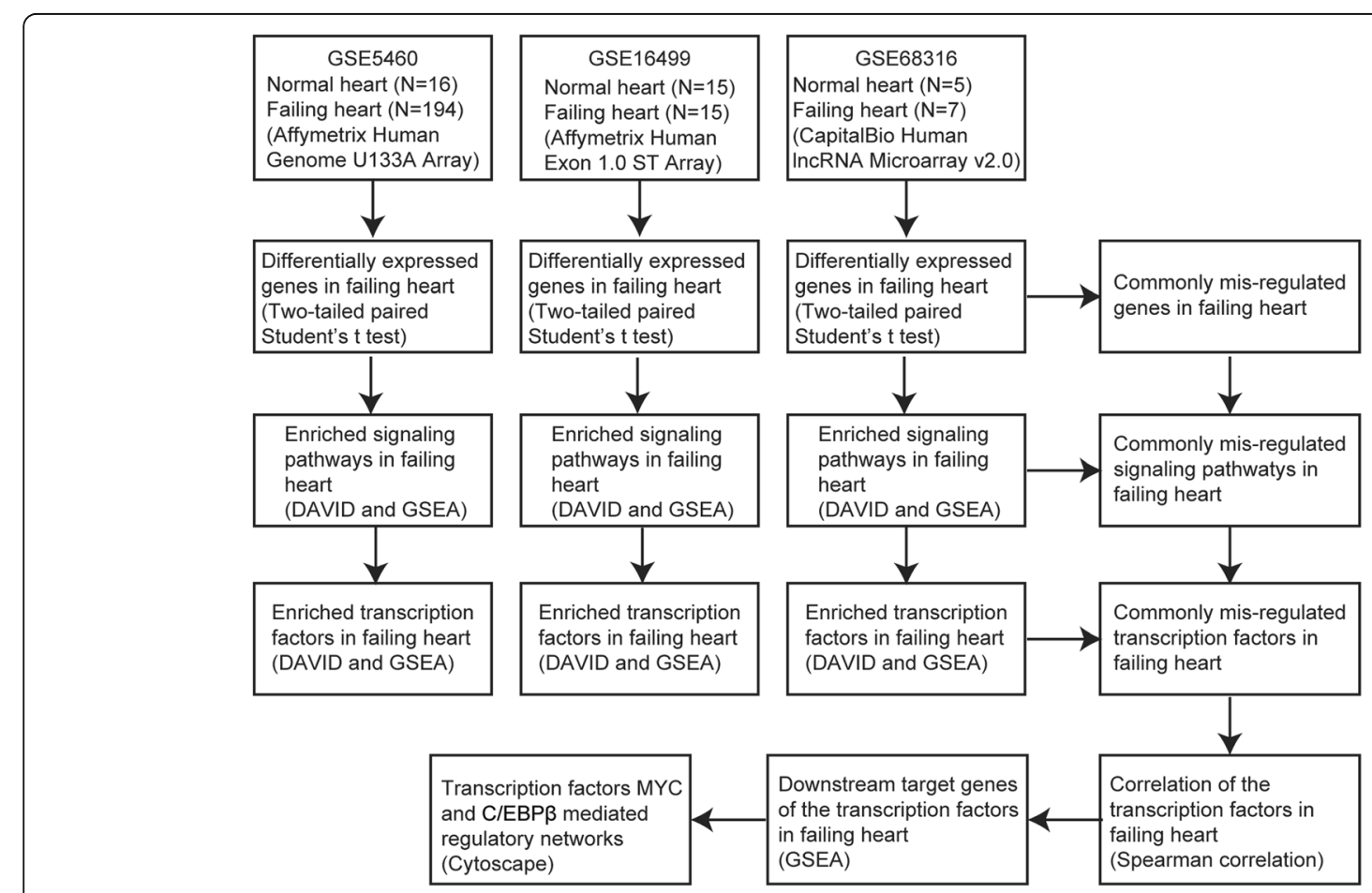

Fig. 1 Search strategies used for accessing the gene datasets was described in the flowchart 
a

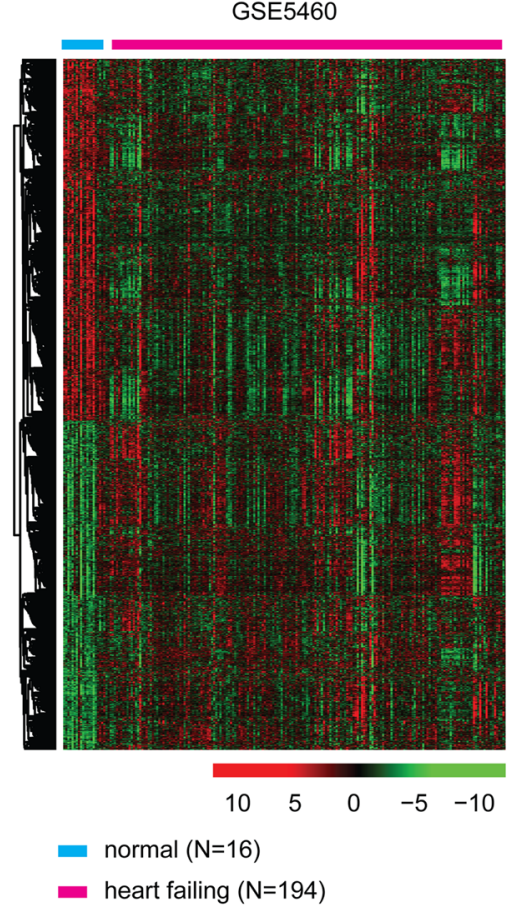

b

heart failing up regulated genes

GSE5460 GSE16499

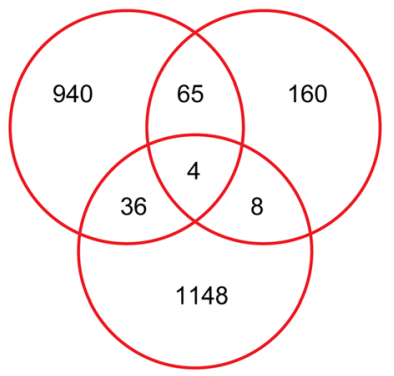

GSE68316
GSE16499

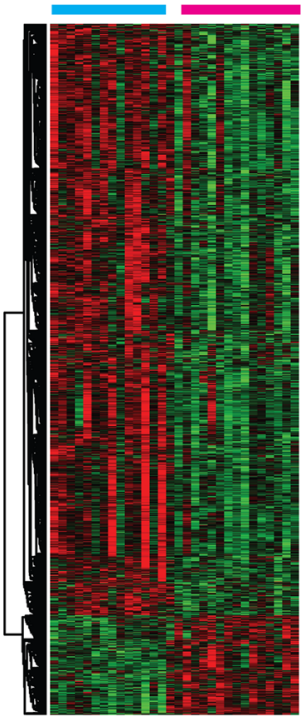

$\begin{array}{lllll}4 & 2 & 0 & -2 & -4\end{array}$

- normal $(\mathrm{N}=15)$

- heart failing $(\mathrm{N}=15)$
GSE68316

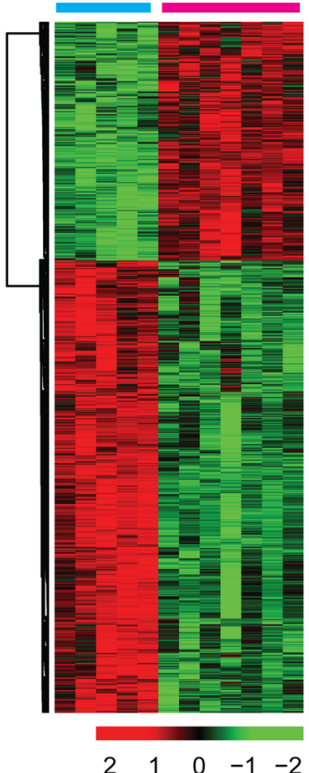

- normal $(\mathrm{N}=5)$

- heart failing $(\mathrm{N}=7)$

heart failing down regulated genes

GSE5460 GSE16499

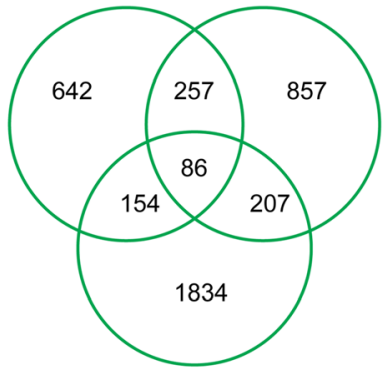

GSE68316

Fig. 2 Identification of the transcriptomic features of heart failure. a Un-supervised clustering heatmaps represented the differentially expressed genes $(P<0.01)$ in failing heart tissues compared to normal heart tissues in GSE5406, GSE16499 and GSE68316 datasets. Each column represented one sample. Genes up-regulated (red), down-regulated (blue) and moderately regulated (black) were delineated. Number of sample in each dataset was also showed. b Venn diagrams depicted the overlapped differentially expressed genes in GSE5406, GSE16499 and GSE68316 datasets. Red cycle represented the up regulated genes in failing heart tissues and green cycle represented the down regulated genes in failing heart tissues

down regulated in GSE5406, GSE16499 and GSE68316 datasets (Fig. 2b). In GSE16499 and GSE68316 datasets, the number of down regulated genes was for more than the up regulated genes (Fig. 2a). In GSE16499 dataset, 1407 genes were suppressed in failing heart tissues. While, only 237 genes were activated in failing heart tissues. Those results suggested that the depletion of cardiomyocytes and loss of mechanical functions in cardiac remodeling were induced by the suppression of heart specific genes.
Metabolism and insulin signaling pathway are suppressed in failing heart patients

To reveal the functional relevance of the common differentially expressed genes in failing heart tissues, we performed functional signaling pathway enrichment analysis through DAVID [22] and GSEA [23] assay. Pyrimidine, purine metabolism signaling pathway and cysteine, methionine metabolism signaling pathway were highly enriched through DAVID analysis (Fig. 3a). Heatmap presentations showed that 


\section{a}

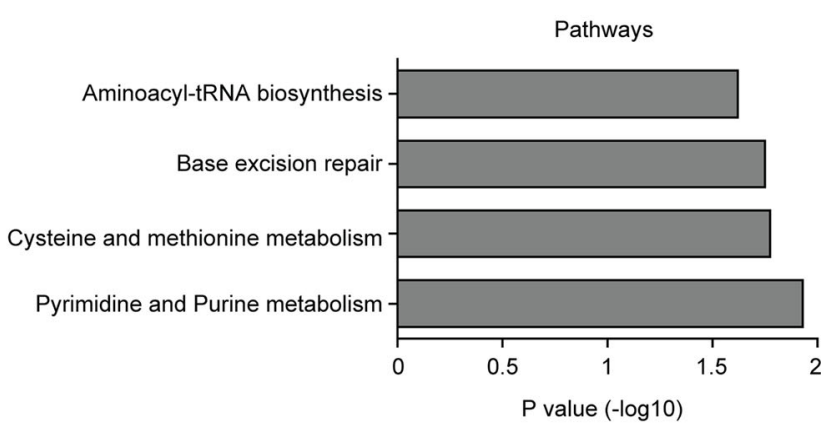

b

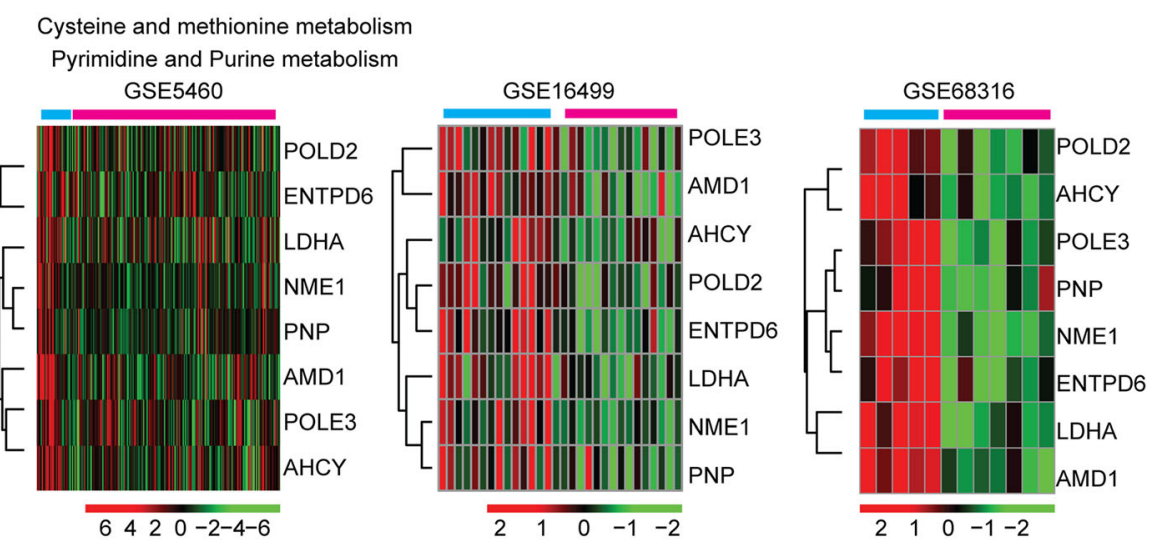

C

Enrichment plot of Insulin signaling pathway

GSE5460
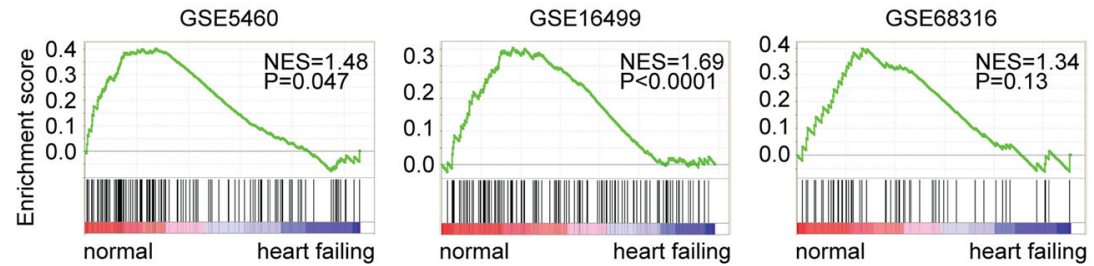

d
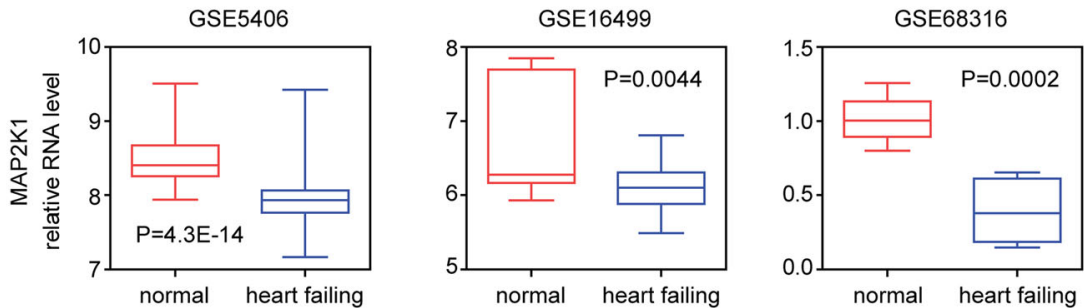

Fig. 3 Metabolism and insulin signaling pathway are suppressed in failing heart patients. a Functional pathways enrichment analysis of the common differentially expressed 90 genes in failing heart tissues. $\mathbf{b}$ Differentially expressed genes from cysteine, methionine, pyrimidine and purine metabolism signaling pathway were represented using heatmaps. c Enrichment plots showed the enriched insulin signaling pathway in GSE5406, GSE16499 and GSE68316 datasets. Enrichment of NES and P values were shown. d Box plots showed the expression levels of MAP2K1 in GSE5406, GSE16499 and GSE68316 datasets. P values showed the difference of MAP2K1 expression levels between failing heart tissues and normal heart tissues determined by Student's t test

NME1, POLE3, POLD2, ENTPD6, PNP genes from pyrimidine, purine metabolism signaling pathway and LDHA, AHCY, AMD1 genes from cysteine, methionine metabolism signaling pathway were all down regulated in failing heart tissues in GSE5406, GSE16499 and GSE68316 datasets (Fig. 3b), suggesting the suppression of those pathways in the development of heart failure. 
Through GSEA analysis, we found that the insulin signaling pathway was negatively correlated with the failing heart expression profiling (Fig. 3c), suggesting the inactivation of insulin signaling pathway in the development of heart failure. Fox example, MAP2K1 is a critical downstream gene of insulin signaling pathway [24]. We showed that MAP2K1 was down regulated in failing heart tissues in GSE5406, GSE16499 and GSE68316 datasets (Fig. 3d).

The association between heart failure, inactivation of metabolism pathways and insulin resistance was well established [24]. The cardiac metabolism, growth and survival in the heart were dependent on insulin signaling pathway [25]. Loss of insulin signaling pathway induced cardiac energy deficiency and accelerated the heart failure progress [26]. All those observations confirmed the enriched singling pathways derived from the GEO datasets.

\section{Transcription factors MYC and C/EBP are negatively} associated with in failing heart expression profiling Except signaling pathways, the transcription factors enriched in failing heart tissues were also identified through DAVID analysis. We found that transcription factor MYC was highly associated with the differentially expressed genes in GSE5406, GSE16499 and GSE68316 datasets (Fig. 4a). Interestingly, TP53 and E2F genes were both highly enriched (Fig. 4a). TP53 and E2F family genes were reported to mediate the cardiac growth and development [27]. However, the functions of MYC in the development of heart failure are unclear.

Similar results were obtained using GSEA assay. We found that transcription factor MYC was negatively associated with the failing heart expression profiling in all three GEO datasets (Fig. 4b). Additionally, we showed that transcription factor C/EBP was also negatively correlated with the failing heart expression profiling (Fig. 4c).

$\mathrm{C} / \mathrm{EBP}$ is a CCAAT/enhancer-binding protein transcription factor which regulates cell growth and differentiation. Previous results showed that C/EBP $\beta$ protected against pathological cardiac remodeling [9]. C/EBP $\beta$ was also a master regulator of metabolism pathways and insulin resistance [12]. All those reports implied the potential roles of $C / E B P \beta$ in the development of heart failure.

\section{Transcription factors MYC and C/EBP $\beta$ are down regulated in failing heart tissues}

Next, we detected the expression of MYC and C/EBP $\beta$ in failing heart and normal heart tissues. Previous report showed that MYC was increased in pathological

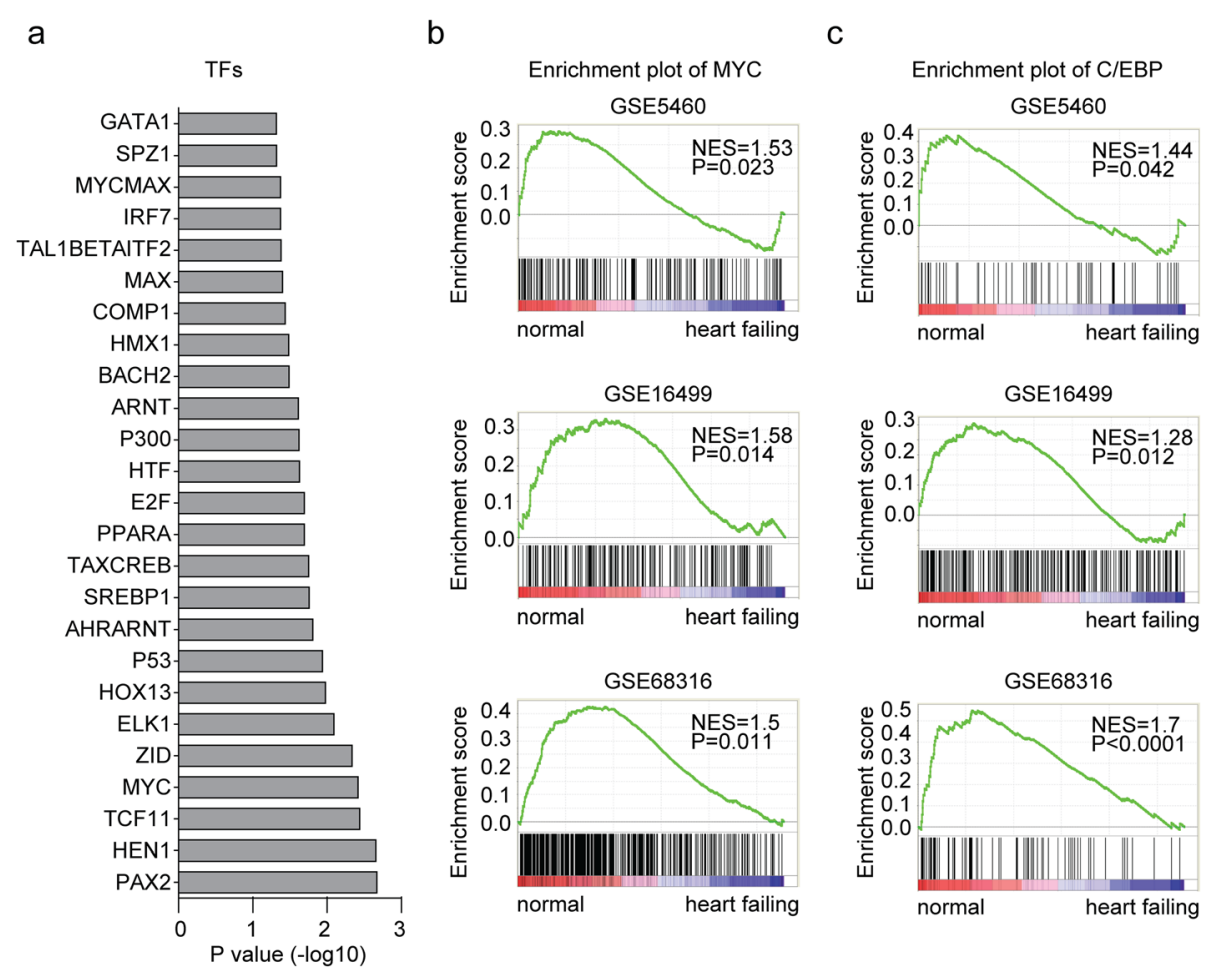

Fig. 4 Transcription factors MYC and C/EBP are negatively associated with in failing heart expression profiling. a Functional transcription factors enrichment analysis of the common differentially expressed 90 genes in failing heart tissues. $\mathbf{b}$ Enrichment plots of transcription factor MYC in GSE5406, GSE16499 and GSE68316 datasets. Enrichment of NES and P values were shown. c Enrichment plots of transcription factor C/EBP in GSE5406, GSE16499 and GSE68316 datasets 
hypertrophy [28]. Inhibition of MYC was a potential therapeutic approach in the treatment of hypertrophic cardiomyopathy [29]. On the contrary, we found the down regulation of MYC expression in failing heart tissues in GSE5406 and GSE16499 datasets (Fig. 5a). Similarly, we found that $\mathrm{C} / \mathrm{EBP} \beta$ was down regulated in failing heart tissues, compared with normal heart tissues in all GSE5406, GSE16499 and GSE68316 datasets (Fig. 5b).

Since MYC and C/EBP $\beta$ were both down regulated in failing heart tissues, we tested the correlation between MYC and C/EBP $\beta$ expression in GSE5406 and GSE16499 datasets. We found that C/EBP $\beta$ expression was positively correlated with MYC expression. Heart tissues with high $\mathrm{C} / \mathrm{EBP} \beta$ expression were also with high MYC expression (Fig. 5c). All those results emphasized the important roles of MYC and C/EBP $\beta$ in heart failure development.

\section{MYC and C/EBP $\beta$ target genes are down regulated in failing heart tissues}

Transcription factors are usually the master regulators of disease and regulate multiple target genes. In the GSEA assay, we identified 62 MYC target genes and 22 $\mathrm{C} / \mathrm{EBP} \beta$ target genes. Consistent with the decreased expressions of MYC and C/EBP $\beta$ in failing heart tissues, MYC target genes were down regulated in failing heart tissues, compared with normal heart tissues (Fig. 6a). C/ EBP $\beta$ target genes were also suppressed in failing heart tissues in GSE16499 dataset, as demonstrated in the heatmap (Fig. 6b).

Interestingly, we found that some genes, for example, EIF4A1, SYNCRIP, ARF6 and C/EBP $\beta$, were both MYC and $C / E B P \beta$ downstream target genes (Fig. 6c). We showed that SYNCRIP gene expression was particularly down regulated in failing heart tissues in all GSE5406, GSE16499 and GSE68316 datasets (Fig. 6d). a

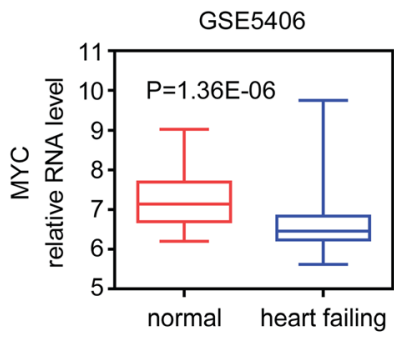

b

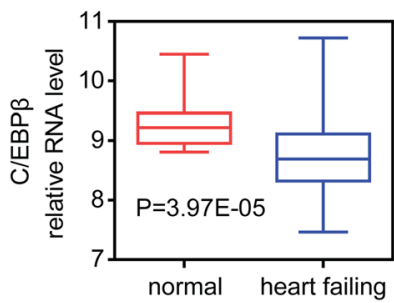

GSE16499

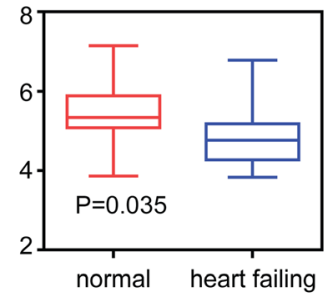

GSE16499

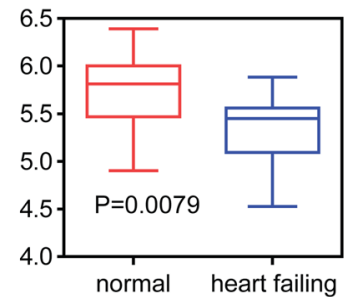

GSE68316

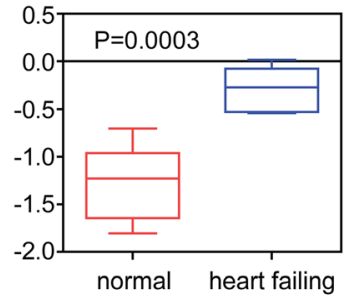

GSE68316

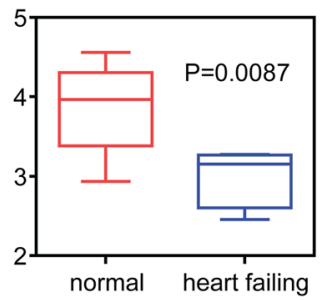

C
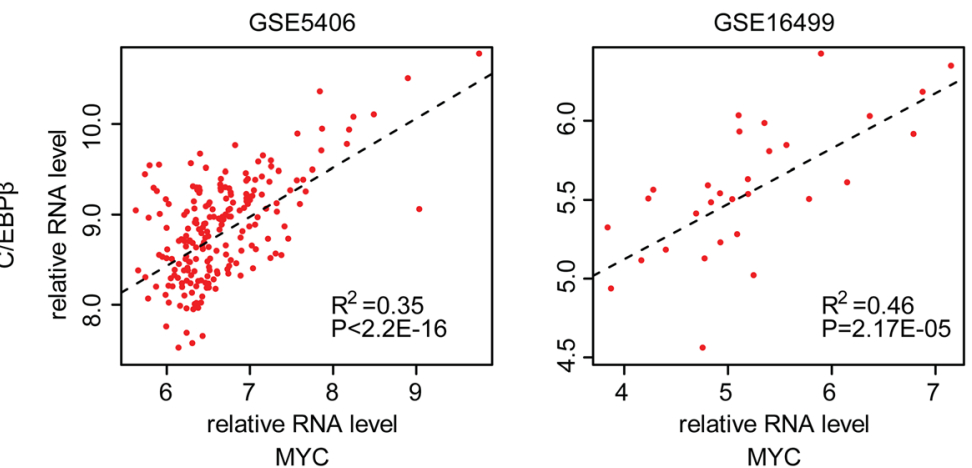

Fig. 5 Transcription factors MYC and C/EBP $\beta$ are down regulated in failing heart tissues. a Box plots showed the transcription factor MYC expression levesl. P values showed the difference of genes expression levels between failing heart and normal heart tissues determined by Student's t test. $\mathbf{b}$ Box plots showed the transcription factor C/EBP $\beta$ expression levels. $\mathbf{c}$ Pearson correlation between MYC and C/EBP $\beta$ expression levels in GSE5406 and GSE16499 datasets. Adjusted R-square and $P$ value were shown 
a

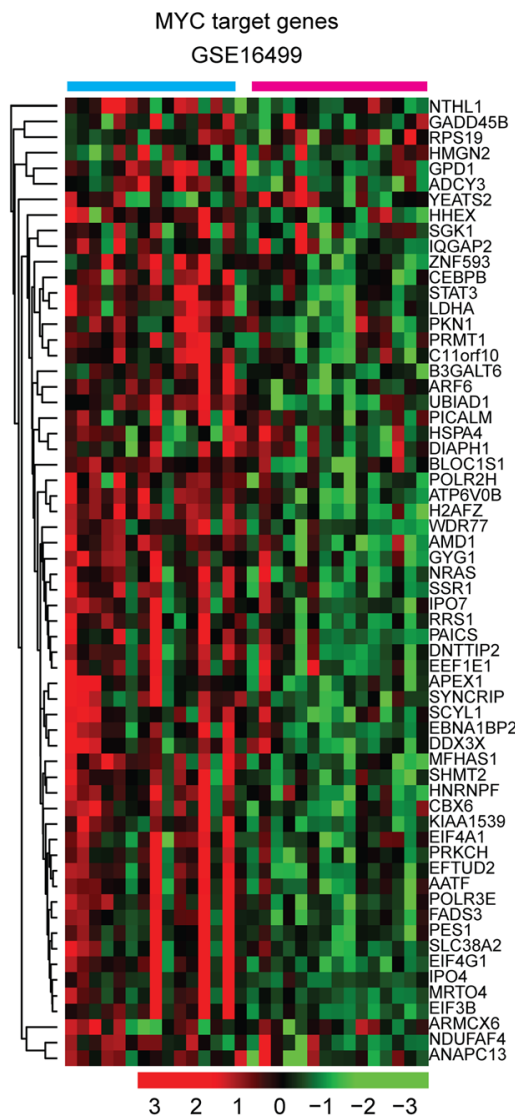

- normal $(\mathrm{N}=15)$

- heart failing $(\mathrm{N}=15)$

d

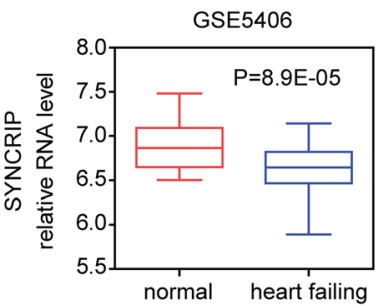

b

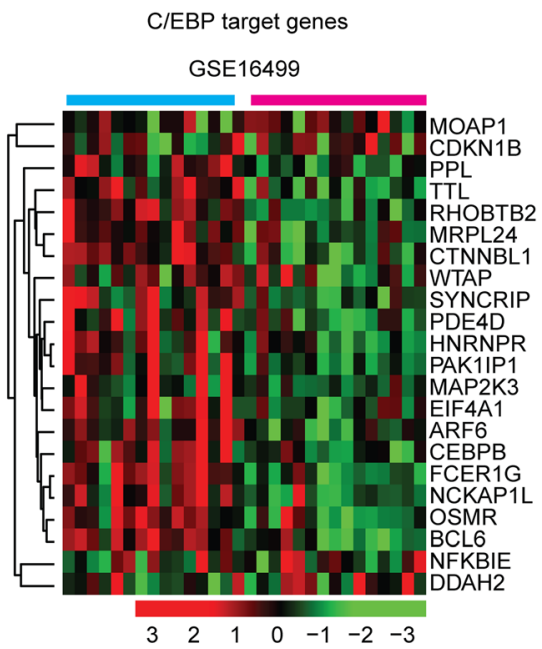

$$
\text { normal }(\mathrm{N}=15)
$$

C

C/EBP target genes

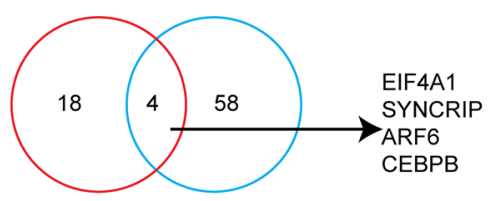

MYC target genes
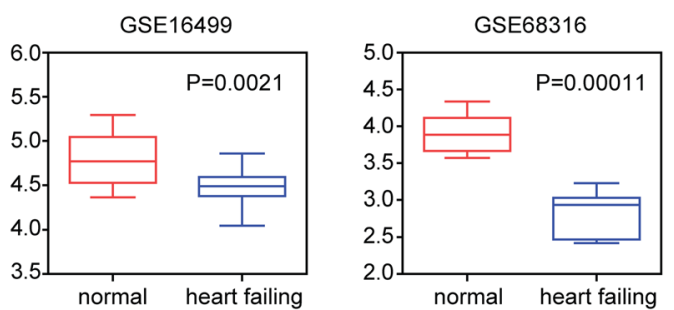

Fig. 6 MYC and C/EBP $\beta$ target genes are down regulated in failing heart tissues. a Heatmap demonstrated the expression profile of MYC target genes in failing heart and normal heart tissues in GSE16499 dataset. Genes up-regulated (red), down-regulated (blue) and moderately regulated (black) were delineated. $\mathbf{b}$ Heatmap demonstrated the expression profile of C/EBP $\beta$ target genes in failing heart and normal heart tissues in GSE16499 dataset. c Venn diagram depicted the four overlapped MYC and C/EBP $\beta$ target genes. $\mathbf{d}$ Box plots showed the expression levels of SYNCRIP

The MYC and C/EBP $\beta$ mediated transcriptional networks To further explore MYC and its connection to downstream target genes, the MYC mediated regulatory network was constructed using Cytoscape. As expected, as a MYC target gene, C/EBP $\beta$ was connected with MYC through the transduction of multiple genes (Fig. 7a). Furthermore, through literature research, we found that some MYC target genes were previously reported involving the development of heart failure, including STAT3 [30], PRMT1 [31], PRKCH [32] and HSPA4 [33] (Fig. 7a).
Similarly, the C/EBP $\beta$ mediated regulatory network was constructed (Fig. 7b). Some C/EBP $\beta$ target genes, for example, OSMR [34], MAP2K3 [35] and CDKN1B [36] also have been studied in heart failure developmental progress (Fig. 7b). All those results highlighted the importance of $\mathrm{MYC}, \mathrm{C} / \mathrm{EBP} \beta$ and their downstream target genes in heart failure development. The functions of other MYC and C/EBP $\beta$ target genes should be further studied to reveal their connections with heart failure. 
a

MYC mediated network

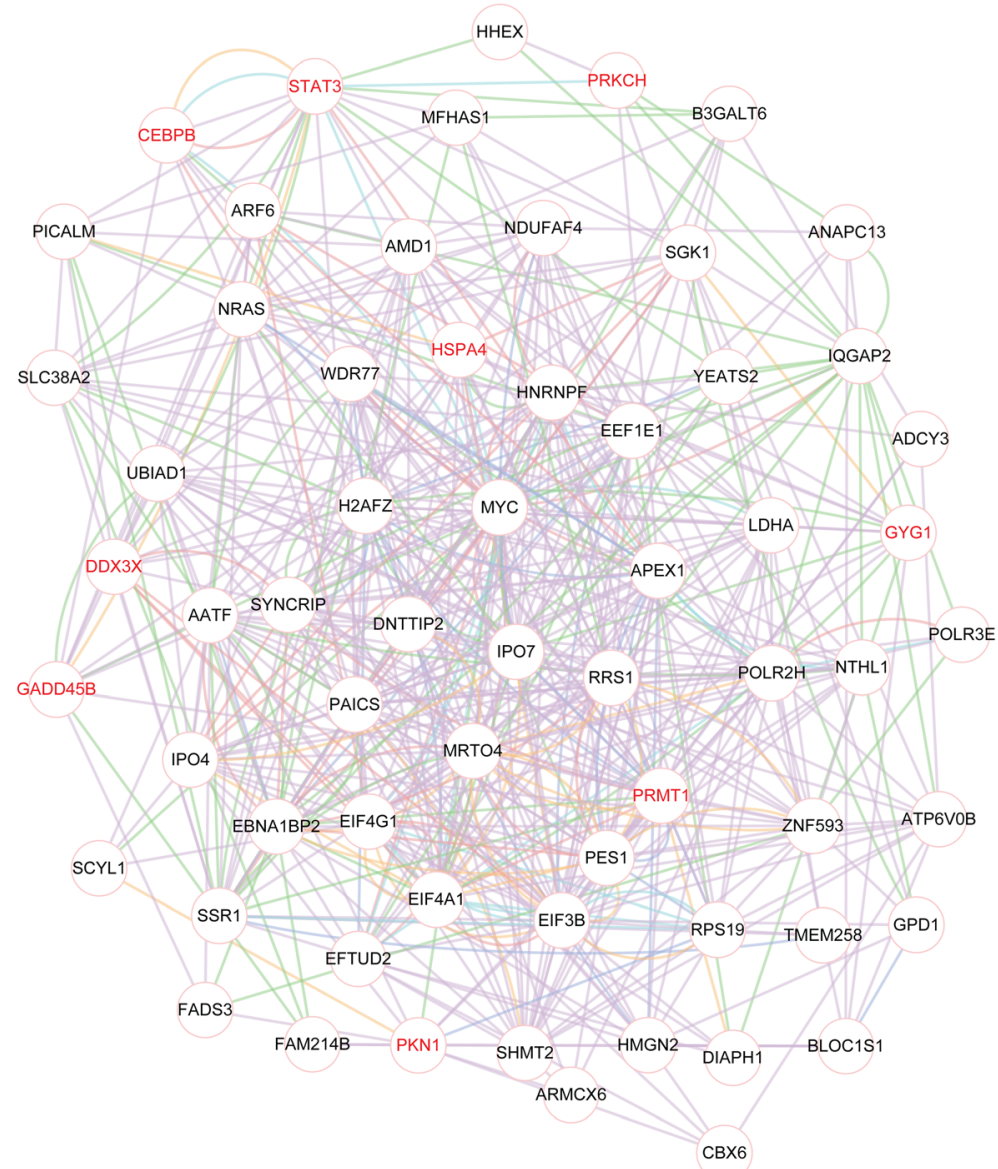

b

CEBPB mediated network

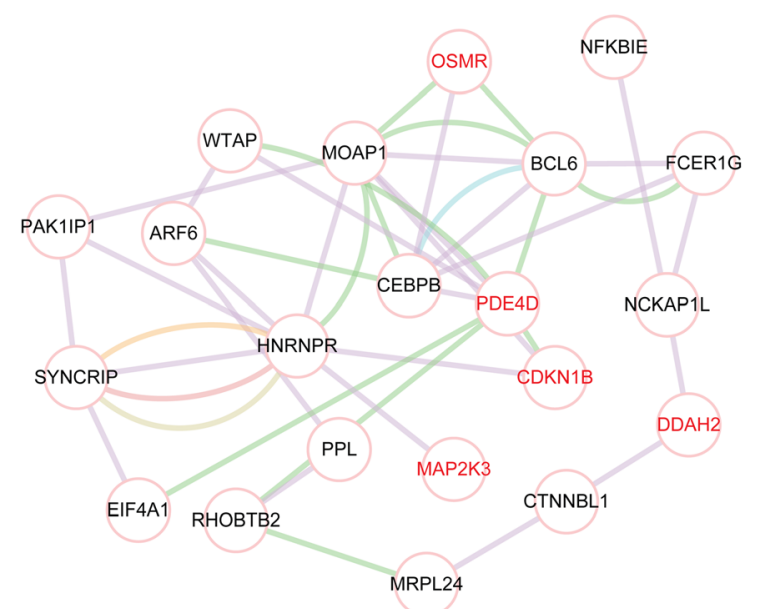

Fig. 7 The MYC and C/EBP $\beta$ mediated transcriptional networks. a MYC mediated regulatory network was created by cytoscape using MYC target genes. The red ones implied that those genes were previously reported involving the development of heart failure. $\mathbf{b} C / E B P \beta$ mediated regulatory network was created by cytoscape using C/EBP $\beta$ target genes

\section{Discussion}

Complex diseases like heart failure are often involving malfunctions of multiple genes. Disease related genes detected by different microarray studies are often highly inconsistent, even when there is not much technical noise [7]. As described in the present study, compared 
with normal heart tissues, there are 2184 differentially expressed genes in failing heart tissues in GSE5406, 1644 genes in GSE16499 and 3477 genes in GSE68316 dataset. However, only 90 genes are commonly up/down regulated in all three datasets. Those differentially expressed genes are associated with MYC and C/EBP $\beta$ transcription factors, metabolism signaling pathways and insulin signaling pathway. The converged transcription factors or signaling pathways may have particularly significant roles in heart failure development than single gene.

Indeed, $C / E B P \beta, M Y C$ and their target genes are all down regulated in failing heart tissues. $C / E B P \beta$ is a master regulator in the development of heart failure [9]. Also, some C/EBP $\beta$ target genes, for example, OSMR [34], MAP2K3 [35] regulate the heart failure developmental progress. The functions of MYC in the regulating of heart failure development are rather complicated. Previous report suggests that inhibition of MYC is a potential therapeutic approach in the treatment of hypertrophic cardiomyopathy [29]. However, we observe the down regulation of MYC expression in failing heart tissues. MYC target genes are also decreased in failing heart tissues. The inconsistence further emphases the complex transcriptional network regulated by MYC and the complex developmental progress of heart failure.

The aim of the current study is to identify the molecular signaling pathways and transcription factors involving failing heart development. By comparative analysis, our results provide the changed expression profiling of metabolism signaling pathway, insulin signaling pathway, transcription factors MYC and C/EBP $\beta$ in the development of heart failure. However, there are certain limitations to the current study. The conclusions were drawn from published databases and lack of further functional validation in failing heart tissues. Therefore, quantitative PCR would have been performed to validate the enriched MYC and C/EBP $\beta$ genes in failing heart tissues. Furthermore, the precise mechanisms of MYC and $\mathrm{C} /$ $\mathrm{EBP} \beta$ in heart failure development require further elucidation by MYC and C/EBP $\beta$ knockout mouse. Nevertheless, our analysis suggests that transcription factor MYC and $C / E B P \beta$ play critical roles in heart failure developmental progress.

\section{Conclusions}

Metabolism signaling pathway, insulin signaling pathway, transcription factors MYC and C/EBP $\beta$ were inhibited in heart failure developmental progress.

\section{Abbreviations}

GEO: Gene expression omnibus; KEGG: Kyoto encyclopedia of gens and genomes; DAVID: The database for annotation, visualization and integrated discovery; GSEA: Gene set enrichment analysis; FDR: False discovery rate

\section{Acknowledgements}

We appreciated the kind supports from Ji Zhang (Ruijin Hospital, Shanghai Jiao Tong University School of Medicine). We acknowledged the working group of R software.

\section{Authors' contributions}

HW.W and XR.W designed and performed data analysis. LP.X helped with the data analysis. HW.W wrote the manuscript. $\mathrm{HC}$ reviewed the manuscript and supervised the work. All listed authors have read and approved the manuscript.

\section{Funding}

This study was supported by grants from Fujian Maternity and Child Health Hospital (No.YCXB 18-10 and YCXM 19-04). But, the funders had no role in study design, data collection and analysis, decision to publish, or preparation of the manuscript.

\section{Availability of data and materials}

The datasets used and analyzed during the current study are available from the corresponding author on reasonable request. Accession numbers of the datasets used in current study are GSE5406, GSE16499 and GSE68316 in Gene Expression Omnibus.

Ethics approval and consent to participate Not applicable.

\section{Consent for publication}

Not applicable.

\section{Competing interests}

The authors declare that they have no competing interests.

Received: 31 October 2019 Accepted: 13 May 2020

Published online: 27 May 2020

\section{References}

1. Vos T, Flaxman AD, Naghavi M, Lozano R, Michaud C, Ezzati M, Shibuya K, Salomon JA, Abdalla S, Aboyans V, et al. Years lived with disability (YLDs) for 1160 sequelae of 289 diseases and injuries 1990-2010: a systematic analysis for the global burden of disease study 2010. Lancet. 2012;380(9859):2163-96.

2. Hashimoto H, Olson EN, Bassel-Duby R. Therapeutic approaches for cardiac regeneration and repair. Nat Rev Cardiol. 2018;15(10):585-600.

3. Lietz K, Miller LW. Improved survival of patients with end-stage heart failure listed for heart transplantation: analysis of organ procurement and transplantation network/U.S. united network of organ sharing data, 1990 to 2005. J Am Coll Cardiol. 2007;50(13):1282-90.

4. Hannenhalli S, Putt ME, Gilmore JM, Wang J, Parmacek MS, Epstein JA, Morrisey EE, Margulies KB, Cappola TP. Transcriptional genomics associates FOX transcription factors with human heart failure. Circulation. 2006;114(12): 1269-76.

5. Kong SW, Hu YW, Ho JW, Ikeda S, Polster S, John R, Hall JL, Bisping E, Pieske B, dos Remedios CG, et al. Heart failure-associated changes in RNA splicing of sarcomere genes. Circ Cardiovasc Genet. 2010;3(2):138-46.

6. Yang W, Li Y, He F, Wu H. Microarray profiling of long non-coding RNA (IncRNA) associated with hypertrophic cardiomyopathy. BMC Cardiovasc Disord. 2015;15:62

7. Li Y, Jiang Q, Ding Z, Liu G, Yu P, Jiang G, Yu Z, Yang C, Qian J, Jiang H, et al. Identification of a Common Different Gene Expression Signature in Ischemic Cardiomyopathy. Genes (Basel). 2018;9(1):56.

8. Bushweller $\mathrm{JH}$. Targeting transcription factors in cancer - from undruggable to reality. Nat Rev Cancer. 2019;19(11):611-24.

9. Bostrom P, Mann N, Wu J, Quintero PA, Plovie ER, Panakova D, Gupta RK, Xiao C, MacRae CA, Rosenzweig A, et al. C/EBPbeta controls exerciseinduced cardiac growth and protects against pathological cardiac remodeling. Cell. 2010;143(7):1072-83.

10. Das B, Young D, Vasanji A, Gupta S, Sarkar S, Sen S. Influence of p53 in the transition of myotrophin-induced cardiac hypertrophy to heart failure. Cardiovasc Res. 2010;87(3):524-34.

11. Nomura S, Satoh M, Fujita T, Higo T, Sumida T, Ko T, Yamaguchi T, Tobita T, Naito AT, Ito M, et al. Cardiomyocyte gene programs encoding 
morphological and functional signatures in cardiac hypertrophy and failure. Nat Commun. 2018:9(1):4435.

12. Takagi M, Uno H, Nishi R, Sugimoto M, Hasegawa S, Piao J, Ihara N, Kanai S, Kakei S, Tamura Y, et al. ATM regulates adipocyte differentiation and contributes to glucose homeostasis. Cell Rep. 2015;10(6):957-67.

13. Vousden KH, Ryan KM. p53 and metabolism. Nat Rev Cancer. 2009;9(10): 691-700.

14. Gomis RR, Alarcon C, Nadal C, Van Poznak C, Massague J. C/EBPbeta at the core of the TGFbeta cytostatic response and its evasion in metastatic breast cancer cells. Cancer Cell. 2006;10(3):203-14.

15. Lopez-Diaz FJ, Gascard P, Balakrishnan SK, Zhao J, Del Rincon SV, Spruck C, Tlsty TD, Emerson BM. Coordinate transcriptional and translational repression of p53 by TGF-beta1 impairs the stress response. Mol Cell. 2013; 50(4):552-64.

16. Gabay M, Li Y, Felsher DW. MYC activation is a hallmark of cancer initiation and maintenance. Cold Spring Harb Perspect Med. 2014;4(6):a014241.

17. Thai M, Thaker SK, Feng J, Du Y, Hu H, Ting Wu T, Graeber TG, Braas D, Christofk HR. MYC-induced reprogramming of glutamine catabolism supports optimal virus replication. Nat Commun. 2015;6:8873.

18. Koh CM, Bezzi M, Low DH, Ang WX, Teo SX, Gay FP, Al-Haddawi M, Tan SY, Osato M, Sabo A, et al. MYC regulates the core pre-mRNA splicing machinery as an essential step in lymphomagenesis. Nature. 2015;523(7558): 96-100

19. Delmore JE, Issa GC, Lemieux ME, Rahl PB, Shi J, Jacobs HM, Kastritis E, Gilpatrick T, Paranal RM, Qi J, et al. BET bromodomain inhibition as a therapeutic strategy to target c-Myc. Cell. 2011;146(6):904-17.

20. Anand P, Brown JD, Lin CY, Qi J, Zhang R, Artero PC, Alaiti MA, Bullard J, Alazem K, Margulies KB, et al. BET bromodomains mediate transcriptional pause release in heart failure. Cell. 2013;154(3):569-82.

21. Duan $Q$, McMahon $S$, Anand $P$, Shah $H$, Thomas $S$, Salunga HT, Huang $Y$, Zhang R, Sahadevan A, Lemieux ME, et al. BET bromodomain inhibition suppresses innate inflammatory and profibrotic transcriptional networks in heart failure. Sci Transl Med. 2017;9(390):eaah5084.

22. Huang da W, Sherman BT, Lempicki RA. Systematic and integrative analysis of large gene lists using DAVID bioinformatics resources. Nat Protoc. 2009; 4(1):44-57.

23. Subramanian A, Tamayo P, Mootha VK, Mukherjee S, Ebert BL, Gillette MA, Paulovich A, Pomeroy SL, Golub TR, Lander ES, et al. Gene set enrichment analysis: a knowledge-based approach for interpreting genome-wide expression profiles. Proc Natl Acad Sci U S A. 2005;102(43):15545-50.

24. Xu J, Keeton AB, Franklin JL, Li X, Venable DY, Frank SJ, Messina JL. Insulin enhances growth hormone induction of the MEK/ERK signaling pathway. J Biol Chem. 2006;281(2):982-92.

25. Bertero E, Maack C. Metabolic remodelling in heart failure. Nat Rev Cardiol, 2018;15(8):457-70.

26. Boudina S, Bugger H, Sena S, O'Neill BT, Zaha VG, Ilkun O, Wright JJ, Mazumder PK, Palfreyman E, Tidwell TJ, et al. Contribution of impaired myocardial insulin signaling to mitochondrial dysfunction and oxidative stress in the heart. Circulation. 2009:119(9):1272-83.

27. King JC, Moskowitz IP, Burgon PG, Ahmad F, Stone JR, Seidman JG, Lees JA. E2F3 plays an essential role in cardiac development and function. Cell Cycle. 2008; (23):3775-80.

28. Trenerry MK, Carey KA, Ward AC, Cameron-Smith D. STAT3 signaling is activated in human skeletal muscle following acute resistance exercise. J Appl Physiol (1985). 2007:102(4):1483-9.

29. Wolfram JA, Lesnefsky EJ, Hoit BD, Smith MA, Lee HG. Therapeutic potential of c-Myc inhibition in the treatment of hypertrophic cardiomyopathy. Ther Adv Chronic Dis. 2011;2(2):133-44.

30. Patel NJ, Nassal DM, Greer-Short AD, Unudurthi SD, Scandling BW, Gratz D, Xu X, Kalyanasundaram A, Fedorov W, Accornero F et al. betalV-Spectrin/ STAT3 complex regulates fibroblast phenotype, fibrosis, and cardiac function. JCl Insight. 2019;4(20).

31. Pyun JH, Kim HJ, Jeong MH, Ahn BY, Vuong TA, Lee DI, Choi S, Koo SH, Cho H, Kang JS. Cardiac specific PRMT1 ablation causes heart failure through CaMKII dysregulation. Nat Commun. 2018;9(1):5107.

32. Ji F, Liu Q, Feng Z, Han X, Li Z. Genetic association between 1425G/a SNP in PRKCH and hypertrophic cardiomyopathy in a Chinese population. Oncotarget. 2017;8(70):114839-44.

33. Mohamed BA, Barakat AZ, Zimmermann WH, Bittner RE, Muhlfeld C, Hunlich M, Engel W, Maier LS, Adham IM. Targeted disruption of Hspa4 gene leads to cardiac hypertrophy and fibrosis. J Mol Cell Cardiol. 2012;53(4):459-68.
34. Hu J, Zhang L, Zhao Z, Zhang M, Lin J, Wang J, Yu W, Man W, Li C, Zhang R, et al. OSM mitigates post-infarction cardiac remodeling and dysfunction by up-regulating autophagy through Mst1 suppression. Biochim Biophys Acta Mol basis Dis. 2017:1863(8):1951-61.

35. Li Y, Li Z, Zhang C, Li P, Wu Y, Wang C, Bond Lau W, Ma XL, Du J. Cardiac fibroblast-specific activating transcription factor 3 protects against heart failure by suppressing MAP2K3-p38 signaling. Circulation. 2017;135(21): 2041-57.

36. Sun X, Momen A, Wu J, Noyan H, Li R, von Harsdorf R, Husain M. p27 protein protects metabolically stressed cardiomyocytes from apoptosis by promoting autophagy. J Biol Chem. 2014;289(24):16924-35.

\section{Publisher's Note}

Springer Nature remains neutral with regard to jurisdictional claims in published maps and institutional affiliations.
Ready to submit your research? Choose BMC and benefit from:

- fast, convenient online submission

- thorough peer review by experienced researchers in your field

- rapid publication on acceptance

- support for research data, including large and complex data types

- gold Open Access which fosters wider collaboration and increased citations

- maximum visibility for your research: over $100 \mathrm{M}$ website views per year

At BMC, research is always in progress.

Learn more biomedcentral.com/submissions 